\title{
AN IMPROVED VERSION OF THE NONCOMPACT WEAK CANONICAL SCHOENFLIES THEOREM
}

\author{
BY
}

W. R. BRAKES

\begin{abstract}
The main result of this paper is that any proper collared embedding of $R^{n-1}$ in $R^{n}$ can be extended to a homeomorphism of $R^{n}$ such that the extension depends continuously on the original embedding in a stronger sense than previously known. Analogous results are proved for proper embeddings of $R^{k}$ in $R^{n}$ (with the usual homotopy conditions when $k=n-2$ ). An alternative proof of the usual compact form of the weak canonical Schoenflies theorem is also obtained.
\end{abstract}

The main problem being considered here may be stated as follows: Given a proper embedding $f$ of $R^{n-1} \times[0,1]$ into $R^{n}$, the generalised Schoenflies theorem (M. Brown [2] and Mazur and Morse [3], [4]) asserts that $f \mid R^{n-1} \times 0$ can be extended to a proper embedding of $R^{n-1} \times[0, \infty)$ into $R^{n}$; can this extension be chosen to vary continuously with respect to $f$ ?

A. Smith [5] provided an affirmative answer to this question, if the topologies on the function spaces are chosen wisely, and conjectured that his theorem could be strengthened. One of the two versions proved here, using somewhat easier methods, is the conjecture of A. Smith, while the other is an alternative stronger form of his theorem.

Following [5], define three topologies on a function space $F(X, Y)$ where $Y$ is metric, a general map $f: X \rightarrow Y$ having a base of neighbourhoods as indicated.

1. The compact-open topology:

$$
U(f, C, \epsilon)=\{g \in F(X, Y) \mid d(f(x), g(x))<\epsilon \text { for all } x \in C\}
$$

for $C$ compact, and $\epsilon>0$.

2. The uniform topology:

$$
U(f, \epsilon)=\{g \in F(X, Y) \mid d(f(x), g(x))<\epsilon \text { for all } x \in X\}
$$

where $\epsilon>0$.

3. The majorant, or fine $C^{0}$ topology:

Received by the editors September 19, 1973 and, in revised form, March 15, 1974.

AMS (MOS) subject classifications (1970). Primary 57A50, 57A45, 57A35; Secondary 57 A15.

Key words and phrases. Euclidean space, proper locally-flat embedding, canonical extension, compact-open topology, uniform topology, majorant topology. 


$$
U(f, \epsilon)=\{g \in F(X, Y) \mid d(f(x), g(x))<\epsilon(x) \text { for all } x \in X\}
$$

where $\epsilon: X \rightarrow(0, \infty)$ is a continuous function.

These are clearly successively finer topologies which coincide when $X$ is compact.

If $M$ and $N$ are manifolds, let $E(M, N)$ denote the set of proper embeddings of $M$ into $N$, where a map $f: M \rightarrow N$ is proper if $f^{-1}(C)$ is compact whenever $C$ is. Let $R_{+}^{n}$ denote $\left\{\left(x_{1}, x_{2}, \ldots, x_{n}\right) \in R^{n} \mid x_{n} \geqslant 0\right\}$.

THEOREM 1. There exist a neighbourhood $U$ of the inclusion in $E\left(R^{n-1} \times[0,1], R^{n}\right)$ with the uniform topology, and a function $F: U \rightarrow$ $E\left(R_{+}^{n}, R^{n}\right)$ such that $F(f)\left|R^{n-1} \times 0=f\right| R^{n-1} \times 0$ and $F$ is continuous with respect to the following topologies: either

(i) the uniform topology on $U$, and the compact-open topology on $F(U)$, or

(ii) the majorant topology on $U$, and the uniform topology on $F(U)$.

Smith's version of this theorem had the first space equipped with the majorant topology, and the second space with the compact-open topology; he also needed the restriction $n>2$, which the following proof does not need. Case (i) is the A. Smith conjecture.

The main tool used in the proof is the technique developed by the author in [1], but knowledge of that paper is not assumed here.

Proof of TheOREM 1. Let

$$
U=U(i, \delta)=\left\{f \in E\left(R^{n-1} \times[0,1], R^{n}\right) \mid d(f(x), x)<\delta\right\}
$$

where $i$ is the inclusion, and $14 \delta<1$. For sets $X$ and $Y$ in $R^{n}$, write $X<Y$ if $x_{n}<y_{n}$ for all $\left(x_{1}, \ldots, x_{n}\right)$ in $X,\left(y_{1}, \ldots, y_{n}\right)$ in $Y$. Suppose $f \in U$. Then

$$
f\left(R^{n-1} \times 2 \delta\right)<R^{n-1} \times 4 \delta<f\left(R^{n-1} \times 6 \delta\right)<R^{n-1} \times 8 \delta,
$$

and $R^{n-1} \times 12 \delta<f\left(R^{n-1} \times 1\right)$.

Define $\varphi: R^{n} \rightarrow R^{n}$ by $\varphi\left(x_{1}, \ldots, x_{n}\right)=\left(x_{1}, \ldots, x_{n}+4 \delta\right)$. Then

$$
f\left(R^{n-1} \times 6 \delta\right)<\varphi f\left(R^{n-1} \times 6 \delta\right)<R^{n-1} \times 12 \delta<f\left(R^{n-1} \times 1\right) .
$$

So

$$
\varphi f\left(R^{n-1} \times 6 \delta\right) \subset \text { Image } f \text { and } R^{n-1} \times 6 \delta<f^{-1} \varphi f\left(R^{n-1} \times 6 \delta\right) .
$$

In fact, since $d(f, i)<\delta$,

$$
R^{n-1} \times 7 \delta<f^{-1} \varphi f\left(R^{n-1} \times 6 \delta\right) \text { and } R^{n-1} \times 5 \delta<f\left(R^{n-1} \times 6 \delta\right) .
$$

Define $\alpha_{1}: R_{+}^{1} \rightarrow R_{+}^{1}$ to satisfy:

$$
\begin{aligned}
& \alpha_{1}(t)=t \quad \text { if } t \geqslant 7 \delta, \quad \alpha_{1}(6 \delta)=2 \delta, \\
& \begin{array}{ll}
\alpha_{1}(13 \delta / 2)=6 \delta, & \alpha_{1}(0)=0,
\end{array}
\end{aligned}
$$

and extend linearly. 
Define $\alpha_{2}: R_{+}^{1} \rightarrow R_{+}^{1}$ to satisfy

$$
\begin{array}{ll}
\alpha_{2}(t)=t & \text { if } t \leqslant 4 \delta, \\
\alpha_{2}(t)=t+4 \delta & \text { if } t \geqslant 5 \delta,
\end{array}
$$

and extend linearly.

Let $A=R^{n-1} \times[6 \delta, 13 \delta / 2]$, and let $B$ be the closure of the region between $R^{n-1} \times 6 \delta$ and $f^{-1} \varphi f\left(R^{n-1} \times 6 \delta\right)$. Define a homeomorphism $g: A \rightarrow B$ by

$$
g=\left(1 \times \alpha_{1}\right)^{-1} f^{-1}\left(1 \times \alpha_{2}\right) f\left(1 \times \alpha_{1}\right) \text {. }
$$

On $R^{n-1} \times 6 \delta, g=$ identity, and on $R^{n-1} \times 13 \delta / 2, g=f^{-1} \varphi f\left(1 \times \alpha_{1}\right)$.

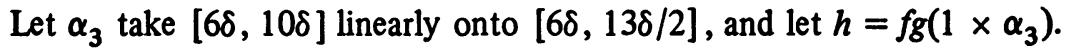
Then $h$ takes $R^{n-1} \times[6 \delta, 10 \delta]$ onto $f(B)$, which is the closure of the region between $f\left(R^{n-1} \times 6 \delta\right)$ and $\varphi f\left(R^{n-1} \times 6 \delta\right)$, and satisfies $h\left|R^{n-1} \times 6 \delta=f\right| R^{n-1} \times$ $6 \delta$ and $\varphi h=h \varphi$ on $R^{n-1} \times 6 \delta$. (See diagram.)

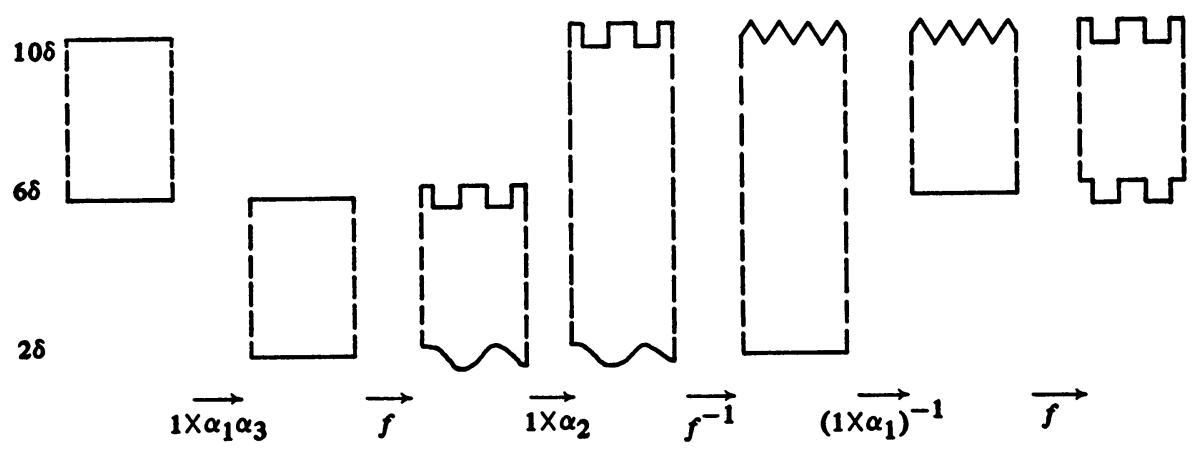

The effect of $h$ on $R^{n-1} \times[6 \delta, 10 \delta]$. The $R^{n-1}$ factor

is drawn horizontally, the $R_{+}^{1}$ factor vertically.

Thus we can extend $h$ to a proper embedding $\bar{h}$ from $R^{n-1} \times[6 \delta, \infty) \rightarrow$ $R^{n}$ as follows: If $x=\left(x_{1}, \ldots, x_{n}\right)$ is in $R^{n-1} \times[6 \delta, \infty)$ there exists $k \geqslant 0$ such that $(4 k+6) \delta \leqslant x_{n}<(4 k+10) \delta$. So $\varphi^{-k}(x) \in R^{n-1} \times[6 \delta, 10 \delta]$, and we can define $\bar{h}(x)=\varphi^{k} h \varphi^{-k}(x)$.

Finally we can extend $\bar{h}$ to a proper embedding $F(f)$ from $R_{+}^{n}$ to $R^{n}$ by setting

$$
\begin{aligned}
F(f)(x) & =f(x) \quad \text { if } 0 \leqslant x_{n} \leqslant 6 \delta \\
& =\bar{h}(x) \text { if } x_{n}>6 \delta
\end{aligned}
$$

Then certainly $F: U \rightarrow E\left(R_{+}^{n}, R^{n}\right)$ and $F(f)\left|R^{n-1} \times 0=f\right| R^{n-1} \times 0$. It remains to check that $F$ is continuous with respect to the topologies of (i) and (ii).

Case (i). Let $V$ be a neighbourhood of $F(f)$ in $E\left(R_{+}^{n}, R^{n}\right)$ with the compactopen topology. Without loss of generality assume $V=U(F(f), C, \epsilon)$ for some com- 
pact $C \subset R_{+}^{n}$ and $\epsilon>0$. There exists a positive real number $N$ such that $C \subset$ $N B^{n-1} \times[0, \infty)$. Then $f \mid(N+1) B^{n-1} \times[0,1]$ and $f^{-1} \mid(N+1) B^{n-1} \times$ $[\delta, 1-\delta]$ are uniformly continuous. Let $\epsilon_{1}$ be such that $d(x, y)<\epsilon_{1}$ and $x$ and $y$ both in $(N+1) B^{n-1} \times[0,1]$ imply that

$$
d(f(x), f(y))<\epsilon / 2 \text {. }
$$

Let $\epsilon_{2}$ be such that $d(x, y)<\epsilon_{2}$ and $x$ and $y$ both in $(N+1) B^{n-1} \times \mathbf{R}^{1} \cap$ (Image $f$ ) imply

$$
d\left(f^{-1}(x), f^{-1}(y)\right)<\epsilon_{1} / 8
$$

Note that

$$
d\left(\alpha_{2}(x), \alpha_{2}(y)\right) \leqslant 5 d(x, y)
$$

and

$$
d\left(\alpha_{1}^{-1}(x), \alpha_{1}^{-1}(y)\right) \leqslant 4 d(x, y)
$$

for all $x$ and $y$.

Let

$$
\epsilon^{\prime}=\operatorname{Min}\left(\epsilon / 2, \epsilon_{1} / 8, \epsilon_{2} / 5\right)
$$

and let $W=U\left(f, \epsilon^{\prime}\right) \cap U$, a neighbourhood of $f$ in $U$ with the uniform topology. Suppose $f^{\prime} \in W$, let $g^{\prime}, h^{\prime}, \vec{h}$ be the corresponding maps constructed from $f^{\prime}$ as above, and suppose $x$ is in $N B^{n-1} \times[6 \delta, 10 \delta]$. Then $\left(1 \times \alpha_{1} \alpha_{3}\right) x \in N B^{n-1} \times$ $[2 \delta, 6 \delta]$. So

$$
d\left(f\left(1 \times \alpha_{1} \alpha_{3}\right) x, f^{\prime}\left(1 \times \alpha_{1} \alpha_{3}\right) x\right)<\epsilon_{2} / 5 \text { (by 5). }
$$

Therefore if $y=\left(1 \times \alpha_{2}\right) f\left(1 \times \alpha_{1} \alpha_{3}\right) x$, and if $y^{\prime}$ is the corresponding expression with $f^{\prime}$ replacing $f$, then $d\left(y, y^{\prime}\right)<\epsilon_{2}$ (by 3), and since both $y$ and $y^{\prime}$ lie in $(N+\delta) B^{n-1} \times R^{1} \cap$ (Image $f$ ),

$$
\left.d\left(f^{-1}(y), f^{-1}\left(y^{\prime}\right)\right)<\epsilon_{1} / 8 \text { (by } 2\right) .
$$

Now if $z=\left(f^{\prime}\right)^{-1}\left(y^{\prime}\right)$, then $z$ is in $(N+2 \delta) B^{n-1} \times[0,1]$. So $f(z)$ and $f^{\prime}(z)\left(=y^{\prime}\right)$ both lie in $(N+1) B^{n-1} \times R^{1} \cap$ (Image $\left.f\right)$. Also

$$
\left.d\left(f(z), f^{\prime}(z)\right)<\epsilon_{2} \text { (by } 5\right) \text {. }
$$

So

$$
\left.d\left(z, f^{-1} f^{\prime}(z)\right)<\epsilon_{1} / 8 \quad \text { (by } 2\right)
$$

That is

$$
d\left(\left(f^{\prime}\right)^{-1}\left(y^{\prime}\right), f^{-1}\left(y^{\prime}\right)\right)<\epsilon_{1} / 8
$$


Combining this with (6) above,

$$
d\left(f^{-1}(y),\left(f^{\prime}\right)^{-1}\left(y^{\prime}\right)\right)<\epsilon_{1} / 4
$$

Therefore

$$
d\left(\left(1 \times \alpha_{1}\right)^{-1} f^{-1}(y),\left(1 \times \alpha_{1}\right)^{-1}\left(f^{\prime}\right)^{-1}\left(y^{\prime}\right)\right)<\epsilon_{1} \quad \text { (by 4). }
$$

That is

$$
d\left(g\left(1 \times \alpha_{3}\right) x, g^{\prime}\left(1 \times \alpha_{3}\right) x\right)<\epsilon_{1}
$$

and since each of these points lies in $(N+2 \delta) B^{n-1} \times[0,1]$,

$$
d\left(f g\left(1 \times \alpha_{3}\right) x, f g^{\prime}\left(1 \times \alpha_{3}\right) x\right)<\epsilon / 2 \quad(\text { by } 1),
$$

and

$$
d\left(f g\left(1 \times \alpha_{3}\right) x, f^{\prime} g^{\prime}\left(1 \times \alpha_{3}\right) x\right)<\epsilon \quad \text { (using 5). }
$$

That is $d\left(h(x), h^{\prime}(x)\right)<\epsilon$.

This is true for all $x$ in $N B^{n-1} \times[6 \delta, 10 \delta]$. So, since $\varphi$ is an isometry, $d(\bar{h}(x), \bar{h}(x))<\epsilon$ for all $x$ in $N B^{n-1} \times[6 \delta, \infty)$ and since $d\left(f, f^{\prime}\right)<\epsilon$,

$$
d\left(F(f)(x), F\left(f^{\prime}\right)(x)\right)<\epsilon \quad \text { for all } x \in N B^{n-1} \times R_{+}^{1} .
$$

Thus $F(W) \subset V$ as required.

Case (ii). Let $V$ be a neighbourhood of $F(f)$ in $E\left(R_{+}^{n}, R^{n}\right)$ with the uniform topology. Without loss of generality assume $V=U(F(f), \epsilon)$ for some $\epsilon>0$.

The proof given above establishes that for any positive integer $N$, there exists $\epsilon_{N}>0$ such that if $d\left(f(y), f^{\prime}(y)\right)<\epsilon_{N}$ for all $y$ in $\left((N+1) B^{n-1}-\operatorname{Int}(N-2) B^{n-1}\right) \times[0,1]$, then $d\left(F(f)(x), F\left(f^{\prime}\right)(x)\right)<\epsilon$ for all $x$ in $\left(N B^{n-1}-\operatorname{Int}(N-1) B^{n-1}\right) \times[0, \infty)$.

Without loss of generality, assume $\epsilon_{M}<\epsilon_{N}$ for all $M>N$, and define $\delta: R^{n-1} \times[0,1] \rightarrow(0, \infty)$ to satisfy $\delta(y)<\epsilon_{N}$ for all $y$ in $\left((N-1) B^{n-1}-\operatorname{Int}(N-2) B^{n-1}\right) \times[0,1]$. Let $W=U(f, \delta) \cap U$, a neighbourhood of $f$ in $U$ with the majorant topology. Then if $f^{\prime} \in W$, and $x \in R_{+}^{n}$, then $x$ is in $\left(N B^{n-1}-\operatorname{Int}(N-1) B^{n-1}\right) \times[0, \infty)$ for some integer $N$. But since $d\left(f(y), f^{\prime}(y)\right)<\delta(y)<\epsilon_{N}$ for all $y$ in $\left((N+1) B^{n-1}-\operatorname{Int}(N-2) B^{n-1}\right) \times[0,1]$ (because $\left.\epsilon_{N+2}<\epsilon_{N+1}<\epsilon_{N}\right), d\left(F(f)(x), F\left(f^{\prime}\right)(x)\right)<\epsilon$. Thus $F(W) \subset V$ as required. This completes the proof of Theorem 1 .

Theorem 1 implies the corresponding noncompact canonical Schoenflies theorem by applying the relevant noncanonical form.

As observed before the statement of Theorem 1, the three topologies introduced there and labelled 1, 2 and 3 are successively finer. Theorem 1 provides an extension that is continuous if the embedding spaces forming the domain and 
the image are given topologies 2 and 1 respectively, or 3 and 2 respectively. It is clearly impossible to get an extension which would be continuous with respect to topologies that gave the image space a finer topology than the domain, but it might be hoped that the same topology could be used for both spaces.

A version of such a canonical extension in Case (1), that is with both spaces equipped with the compact-open topology, is in fact proved in Theorem 1(i) above. However in the "true" version, $U$ (the domain of $F$ ) would be a neighbourhood of the inclusion in the compact-open topology, and such a theorem is clearly impossible: $f$ would have too much freedom outside any given compact set. However there are no such obvious reasons why the other cases should not hold; one might conjecture that extensions as in Theorem 1 could be constructed to be continuous with respect to the original embedding with both spaces equipped with either the uniform topology (2) or with the majorant topology (3).

Failure of the above proof to yield this result in Case (2) can be traced to the fact that if $M$ is a noncompact metric manifold, and $H(M)$ is given the uniform topology, then the function taking $f$ to $f^{-1}$ fails to be continuous at points $f$ for which $f^{-1}$ is not uniformly continuous. The proof given does not work for Case (3) (both spaces having the majorant topology) because the construction cannot be made to "fade out" in the $[0, \infty)$ direction of the extension.

It is clear that the proof given above in Case (i) is equally valid for a general manifold in place of $R^{n-1}, N B^{n-1}$ and $(N+1) B^{n-1}$ being replaced by appropriate compact sets, and so the following theorem is obtained:

THEOREM 2. If $M$ is any metrizable manifold, provide $E\left(M \times[0,1], M \times R^{1}\right)$ with the uniform topology, and $E\left(M \times[0, \infty), M \times R^{1}\right)$ with the compactopen topology. Then there exist a neighbourhood $U$ of the inclusion in $E\left(M \times[0,1], M \times R^{1}\right)$ and a continuous function $F: U \rightarrow E\left(M \times[0, \infty), M \times R^{1}\right)$ such that for all $f$ in $U, F(f)|M \times 0=f| M \times 0$.

In this case of course, Theorem 2 yields no information about neighbourhoods of a general embedding from $M \times[0,1]$ to $M \times R^{1}$.

Setting $M=S^{n-1}$ in Theorem 2, and observing that the natural homeomorphism $S^{n-1} \times R^{1} \rightarrow R^{n}-0$ induces homeomorphisms of the corresponding embedding spaces, recaptures a form of the compact canonical Schoenflies theorem:

THEOREM 3. There exist a neighbourhood $U$ of the inclusion in $E\left(2 B^{n}-\right.$ Int $\left.B^{n}, R^{n}\right)$ and a continuous map $F: U \rightarrow E\left(R^{n}-\right.$ Int $\left.B^{n}, R^{n}\right)$ such that for all $f$ in $U, F(f)\left|S^{n-1}=f\right| S^{n-1}$, each embedding space having the compact-open topology.

As a corollary to Theorem 3, by considering the one-point compactification of $R^{n}$ and inverting, we obtain the usual form of the canonical Schoenflies theorem: 
THEOREM 4. There exist a neighbourhood $U$ of the inclusion in $E\left(B^{n}-1 / 2 B^{n}, R^{n}\right)$ and a continuous map $F: U \rightarrow E\left(B^{n}, R^{n}\right)$ such that $F(f) \mid S^{n-1}$ $=f \mid S^{n-1}$ for all $f$ in $U$.

So far, this work has concentrated on extending embeddings of codimension 1, using the generalised Schoenflies theorem. For codimensions greater than 1, J. Stallings [6] has proved an analogous theorem which can be stated in the following form:

Proposition [6, Theorem 9.2]. If $f: R^{k} \rightarrow R^{n}(n \geqslant 5)$ is a locally flat embedding, with $k \leqslant n-3$, or $k=n-2,\left(R^{n}, f\left(R^{k}\right)\right)$ unravelled at infinity, and $R^{n}-f\left(R^{k}\right)$ having the homotopy type of $S^{1}$, then there exists a homeomorphism $h$ of $R^{n}$ such that $h \mid R^{k}=f$.

It is natural to ask whether this extension can be chosen to vary continuously with respect to $f$ in some sense. Theorem 6 asserts that it can, being a corollary of the following more general theorem. Let $B^{k}$ denote the closed unit ball in $R^{k}$, and if $M$ is any manifold, let $H(M)$ denote the space of self-homeomorphisms of $M$.

Theorem 5. Let $M$ be any metrizable manifold. Provide $E\left(M \times B^{k}, M \times R^{k}\right)$ with the uniform topology, and $H\left(M \times R^{k}\right)$ with the compact-open topology. Then there exist a neighbourhood $U$ of the inclusion in $E\left(M \times B^{k}, M \times R^{k}\right)$ and a continuous function $F: U \rightarrow H\left(M \times R^{k}\right)$ such that $F(f)|M \times 0=f| M \times 0$.

Proof. The proof follows the same lines as that of Theorem 1. Let $U=$ $U(i, \delta)$, a neighbourhood of the inclusion in $E\left(M \times B^{k}, M \times R^{k}\right)$ with the uniform topology. In this case it is simpler to take $\delta$ small enough so that $18 \delta<1$. Suppose $f \in U$. Then

$$
f\left(M \times 2 \delta B^{k}\right) \subset M \times 4 \delta B^{k} \subset f\left(M \times 6 \delta B^{k}\right) \subset M \times 8 \delta B^{k} .
$$

Define $\varphi: M \times R^{k} \rightarrow M \times R^{k}$ by $\varphi(x, t)=(x, 2 t)$.

The proof proceeds as for Theorem 1 with the appropriate linguistic changes.

The new $\alpha_{1}$ is a radial homeomorphism of $R^{k}$ which restricts to the previous $\alpha_{1}$ in each ray. $\alpha_{2}$ is defined by

$$
\begin{aligned}
\alpha_{2}(t) & =t & & \text { if }\|t\| \leqslant 4 \delta, \\
& =2 t & & \text { if }\|t\| \geqslant 5 \delta
\end{aligned}
$$

$\alpha_{3}$ takes $\mathrm{Cl}\left(12 \delta B^{k}-6 \delta B^{k}\right)$ onto $\mathrm{Cl}\left((13 / 2) \delta B^{k}-6 \delta B^{k}\right)$ and is linear on each ray.

[Cl denotes closure.] In this case $g$ is a homeomorphism from $M \times$ $\mathrm{Cl}\left((13 \delta / 2) B^{k}-6 \delta B^{k}\right)$ onto $\mathrm{Cl}\left(M \times 6 \delta B^{k}-f^{-1} \varphi f\left(M \times 6 \delta B^{k}\right)\right)$, and $h=$ $f g\left(1 \times \alpha_{3}\right)$ as before.

$h$ satisfies the following: 
$h\left|M \times 6 \delta S^{k-1}=f\right| M \times 6 \delta S^{k-1}$ and $\varphi h=h \varphi \quad$ on $M \times 6 \delta S^{k-1}$.

Thus $h$ can be extended to a proper embedding $\bar{h}$ from $M \times \mathrm{Cl}\left(R^{k}-6 \delta B^{k}\right)$ into $M \times R^{k}$ as follows:

If $(x, t) \in M \times \mathrm{Cl}\left(R^{k}-6 \delta B^{k}\right)$, then there exists $r \geqslant 0$ such that $2^{r} \cdot 6 \delta \leqslant$ $t<2^{r} \cdot 12 \delta$. So $\varphi^{-r}(x, t) \in M \times \operatorname{Cl}\left(12 \delta B^{k}-6 \delta B^{k}\right)$ and $\bar{h}$ can be defined by $\bar{h}(x, t)=\varphi^{r} h \varphi^{-r}(x, t)$.

Finally $\bar{h}$ can be extended to a homeomorphism $F(f)$ of $M \times R^{k}$ by setting

$$
\begin{aligned}
F(f)(x) & =f(x) \quad \text { if } x \in M \times 6 \delta B^{k}, \\
& =\bar{h}(x) \quad \text { otherwise. }
\end{aligned}
$$

To check continuity of $F$, let $V$ be a neighbourhood of $F(f)$ in $H\left(M \times R^{k}\right)$ with the compact-open topology. Without loss of generality assume $V=$ $U(F(f), C, \epsilon)$ for some compact $C \subset M \times R^{k}$ and $\epsilon>0$. There exist a compact $D \subset M$ and a finite $K$ such that $C \subset D \times K B^{k}$.

The first part of the proof of case (i) of Theorem 1 passes over to this situation, with only the obvious changes, to prove the following:

Given an $\epsilon_{1}>0$, there exists $\epsilon_{2}>0\left(\epsilon_{2}<\epsilon_{1}\right)$ such that if $W=U\left(f, \epsilon_{2}\right)$ $\cap U$, a neighbourhood of $f$ in $U$, and $f^{\prime} \in W$, then $d\left(h(x), h^{\prime}(x)\right)<\epsilon_{1}$ for all $x$ in $D \times \mathrm{Cl}\left(12 \delta B^{k}-6 \delta B^{k}\right)$.

Now suppose $(x, t) \in D \times K B^{k}$. There exists $r$ such that $2^{r} \cdot 6 \delta \leqslant t<$ $2^{r} \cdot 12 \delta$, where $2^{r} \cdot 6 \delta<K$ and

$$
\bar{h}(x, t)=\varphi^{r} h\left(x, t / 2^{r}\right), \quad(\bar{h})^{\prime}(x, t)=\varphi^{r} h^{\prime}\left(x, t / 2^{r}\right) .
$$

If $f^{\prime} \in W$, with $\epsilon_{2}$ corresponding to some (still to be determined) $\epsilon_{1}$, then

$$
d\left(h\left(x, t / 2^{r}\right), h^{\prime}\left(x, t / 2^{r}\right)\right)<\epsilon_{1},
$$

so

$$
d\left(p_{1} \bar{h}(x, t), p_{1}(\bar{h})^{\prime}(x, t)\right)<\epsilon_{1} .
$$

Also

So

$$
d\left(p_{2} \bar{h}(x, t), p_{2}(\bar{h})^{\prime}(x, t)\right)<2^{r} \cdot \epsilon_{1}
$$

$$
d\left(\bar{h}(x, t),(\bar{h})^{\prime}(x, t)\right)<\left(2^{r}+1\right) \epsilon_{1} .
$$

Therefore if $\epsilon_{1}$ is chosen so that $\epsilon_{1}<\epsilon /((K / 6 \delta)+1)$ ( $\delta$ is fixed and $K$ and $\epsilon$ depend only on $V$ ), then

$$
d\left(F(f)(x, t), F\left(f^{\prime}\right)(x, t)\right)<\epsilon \quad \text { for all }(x, t)
$$

in $D \times K B^{k}$ and so $F\left(f^{\prime}\right) \in U\left(F(f), D \times K B^{k}, \epsilon\right) \subset V$. Thus $F(W) \subset V$, and $F$ is continuous. 
Note that if $f$ is piecewise linear in the above theorem then $F(f)$ will be piecewise linear. Also it would not be hard to arrange $F(f)$ to be differentiable if $f$ is differentiable.

As stated above, the most important case of this theorem occurs when $M$ is a Euclidean space, and so for emphasis the following immediate corollary of Theorem 5 is stated explicitly.

THEOREM 6. If $E\left(R^{r} \times B^{k}, R^{r+k}\right)$ has the uniform topology, and $H\left(R^{r+k}\right)$ has the compact-open topology, then there exist a neighbourhood $U$ of the inclusion in $E\left(R^{r} \times B^{k}, R^{r+k}\right)$ and a continuous function $F$ from $U$ into $H\left(R^{r+k}\right)$ such that $F(f)\left|R^{r} \times 0=f\right| R^{r} \times 0$.

Note that in this case the techniques used here do not yield a version of this theorem to correspond with part (ii) of Theorem 1: there is no control over the behaviour of $F(f)$ towards infinity in the $R^{k}$ direction.

For tile same reason the method does not appear strong enough to deduce the corresponding compact version, that is a canonical extension for locally flat embeddings of $S^{k}$ in $S^{n}$, where $n-k>1$.

\section{REFERENCES}

1. W. R. Brakes, k-stability of homeomorphisms of Euclidean n-space, Proc. Cambridge Philos. Soc. 75 (1974), 175.

2. M. Brown, A proof of the generalised Schoenflies theorem, Bull. Amer. Math. Soc. 66 (1960), 74-76. MR 22 \#8470b.

3. B. Mazur, On embeddings of spheres, Bull. Amer. Math. Soc. 65 (1959), 59-65. MR 22 \#8469.

4. M. Morse, A reduction of the Schoenflies extension problem, Bull. Amer. Math. Soc. 66 (1960), 113-115. MR 22 \#8470a.

5. A. Smith, Extending embeddings of $R^{n-1}$ in $R^{n}$, Proc. Cambridge Philos. Soc. 71 (1972), 5-18. MR 44 \#7573.

6. J. R. Stallings, On topologicaily unknotted spheres, Ann. of Math. (2) 77 (1963), 490-503. MR 26 \#6946.

DEPARTMENT OF PURE MATHEMATICS, CAMBRIDGE UNIVERSITY, CAMBRIDGE, ENGLAND

Current address: Department of Mathematics, Ahmadu Bello University, Zaria, Nigeria 